ISSN electrónico: 2445-1355

DOI: https://dx.doi.org/10.14201/fj2020522741

\title{
DETERMINACIÓN DE LA COMPOSICIÓN FENÓLICA EN GRANOS ANCESTRALES DE USO ALIMENTARIO. COMPARACIÓN DE MÉTODOS ANALÍTICOS Y DE ACTIVIDAD ANTIOXIDANTE
}

\author{
Determination of Phenolic Composition in Ancestral \\ Grains for Food Use. Comparison of Analytical Methods \\ and Antioxidant Activity
}

Magdalena M. ${ }^{a}$ DELCOURT-GARCÍA; María Teresa ESCRIBANO-BAILÓN;

Montserrat DUEÑAS

Grupo de Investigación en Polifenoles. Departamento Química Analítica, Nutrición y Bromatología. Facultad de Farmacia. Universidad de Salamanca. España

Correo-e: mduenas@usal.es

RESUMEN: El objetivo de este estudio fue cuantificar los compuestos fenólicos totales de diferentes granos ancestrales: dos variedades de tef (Eragrostis tef), trigo sarraceno (Fagopyrum esculentum) y trigo espelta (Triticum spelta) por diferentes métodos espectrofotométricos (FolinCiocalteu y Fast Blue BB) y comparar con los resultados obtenidos de estudiar la composición fenólica individualizada mediante cromatografía líquida de alta resolución acoplada a un sistema de detección de fotodiodos y espectrometría de masas (HPLC-DAD-MS). También se determinó la actividad antioxidante in vitro de los extractos de los granos ancestrales estudiados por el método TEAC (capacidad antioxidante equivalente de Trolox) y FRAP (poder antioxidante reductor del hierro). El uso de HPLCDAD-MS permitió identificar un gran número de compuestos fenólicos: compuestos hidroxibenzoicos, hidroxicinámicos, flavonoles, flavonas y flavanoles. Las flavonas fueron la familia de compuestos fenólicos más abundante detectada en las muestras de espelta, teff, blanco y marrón. Sin embargo, en el trigo sarraceno son los flavanoles (64\%). La cuantificación

Ediciones Universidad de Salamanca / @@ఠ $\quad$ FarmaJournal, vol. 5, núm. 2 (2020), pp. 27-41 
de los compuestos fenólicos por HPLC-DAD-MS parece más oportuna que por los métodos espectrofotométricos. Con respecto a la actividad antioxidante, el trigo sarraceno mostró los valores más altos frente a su capacidad de captar radicales libres y poder reductor en relación a las otras muestras estudiadas, pudiéndose relacionar con su alto contenido en compuestos fenólicos.

Palabras clave: Compuestos fenólicos; granos ancestrales; Folin-Ciocalteu; Fast-Blue BB; HPLC-DAD-MS; actividad antioxidante.

ABSTRACT: The aim of this study was to quantify the total phenolic compounds of different ancestral grains such as two varieties of tef (Eragrostis tef) white and brown, buchwheat (Fagopyrum esculentum) and spelta (Triticum spelta), using different spectrophotometric methods (Folin-Ciocalteu and Fast Blue BB) and to compare with the obtained results using high performance liquid chromatography coupled to diode array detector and mass spectrometry (HPLC-DAD-MS). Furthermore, antioxidant activity of the extracts from these samples has been compared using two different in vitro assays: ferric reducing power (FRAP) and the ability to scavenge the ABTS ${ }^{\circ}$. The use of HPLC-DAD-MS allowed us to detect different groups of phenolic compounds: hydroxybenzoic and hydroxycinnamic derivatives, flavonols, flavones and flavanols. Flavones derivatives were the most abundant in spelta, white and brown teff. However, buckwheat presented the highest in flavanols (64\%). The phenolic compounds quantification carried out by HPLC-DAD-MS could be more appropriate than the obtained results using spectrophotometric methods. Regarding the antioxidant activity, the buckwheat seeds showed the highest value, which could be related to the highest phenolic content.

Keywords: Phenolic compounds; ancestral grains; Folin-Ciocalteu; Fast-Blue BB; HPLC-DAD-MS; antioxidant activity.

\section{INTRODUCCIÓN}

\subsection{Granos ancestrales: características y composición}

Cada vez existe una mayor conciencia sobre lo que comemos y sobre los efectos que tienen los alimentos sobre la salud. En este contexto, están cobrando cada vez más importancia productos como las semillas ancestrales, por las 
múltiples propiedades nutricionales y efectos beneficiosos que podrían ejercer sobre nuestra salud.

Los granos ancestrales son aquellos que no han sido transformados genéticamente con el paso del tiempo. Algunos de ellos han sido cultivados durante siglos; aunque en ocasiones su cultivo se ha restringido a áreas locales. En la actualidad, estas semillas están emergiendo de nuevo con fuerza debido a sus propiedades nutricionales. Aportan un alto contenido en fibra dietética, vitaminas, minerales, proteínas, almidón resistente.

Algunas de las semillas ancestrales más importantes son teff, trigo sarraceno y trigo espelta. El teff (Eragrostis tef (Zucc.) Trotter) es un cereal anual procedente de Etiopía, perteneciente a la familia Poaceae. Se distinguen principalmente dos variedades, blanco y marrón o rojo. Destaca por su alto contenido en lisina, hierro y calcio (Baye, 2014). El trigo sarraceno (Fagopyrum esculentum Moench) es un pseudocereal procedente de Asia Central, pertenece a la familia Poligonaceae. Es rico en lisina y arginina. También, presenta altos niveles de ácidos grasos poliinsaturados y almidón (Sedeje et al., 2012). El trigo espelta (Triticum spelta) es una variedad de trigo que proviene del Antiguo Egipto, perteneciente a la familia Poaceae. Se caracteriza por su contenido en triptófano y lisina; y su alto contenido en magnesio, hierro, fósforo y zinc (Keriené et al., 2015). También hay que destacar que tanto el teff y como el trigo sarraceno no tienen gluten, lo que permite su uso en dietas para personas celiacas. El teff y la espelta se caracterizan por tener un índice glucémico bajo (Sedeje et al., 2012).

Además de su calidad nutricional, estas semillas presentan compuestos bioactivos, tales como como fitoesteroles, vitaminas, y entre los que destacan los compuestos fenólicos, estando estos últimos estrechamente relacionados con efectos beneficiosos para la salud. Existen evidencias científicas sobre el potencial de los compuestos fenólicos como propiedades antioxidantes, anticancerígenas, cardioprotectoras, antiinflamatorias, antienvejecimiento y antimicrobianas, e incluso una mejora en la microbiota intestinal (Del Rio et al., 2013; Fraga et al., 2019). Los efectos biológicos de los flavonoides se han relacionado con sus propiedades antioxidantes y captadoras de radicales libres.

Los objetivos del trabajo son: i) estudiar la composición fenólica individualizada mediante HPLC-DAD-MS de diferentes granos ancestrales tales como teff blanco, marrón, trigo sarraceno y espelta. ii) Cuantificar los compuestos fenólicos totales de las semillas ancestrales por diferentes métodos espectrofotométricos (Folin-Ciocalteu y Fast Blue BB) y comparar con los resultados obtenidos mediante HPLC-DAD-MS. iii) Determinar la actividad antioxidante in vitro de los extractos de los granos ancestrales estudiados por el método TEAC (capacidad antioxidante equivalente de Trolox) y FRAP (poder antioxidante reductor del hierro). 
MAGDALENA M. ${ }^{a}$ DELCOURT-GARCÍA; MARÍA TERESA ESCRIBANO-BAILÓN; MONTSERRAT DUEÑAS DETERMINACIÓN DE LA COMPOSICIÓN FENÓLICA EN GRANOS ANCESTRALES DE USO ALIMENTARIO. COMPARACIÓN DE MÉTODOS ANALÍTICOS Y DE ACTIVIDAD ANTIOXIDANTE

\section{MATERIALES y MÉTODOS}

\subsection{Muestras}

Semillas ancestrales de trigo sarraceno, dos variedades de teff proporcionadas por la empresa Dacsa-Molendum Ingredients (Zamora) y harina de trigo espelta comercial.

\subsection{Preparación de las muestras y extracción de los compuestos fenólicos}

Los granos de teff blanco, marrón y trigo sarraceno fueron triturados en un molinillo para obtener las harinas correspondientes.

La extracción de los compuestos fenólicos se realizó siguiendo el método de Dueñas et al. (2015). La purificación de los compuestos fenólicos se realizó mediante extracción en fase sólida con cartuchos de Sep Pak C ${ }_{18}$ (Waters). Posteriormente, los compuestos fenólicos fueron analizados por HPLC-DAD-MS, según el método descrito por Dueñas et al. (2015).

\subsection{Análisis de compuestos fenólicos mediante HPLC-DAD-MS}

El análisis de compuestos fenólicos se llevó a cabo utilizando un cromatógrafo Hewlett-Packard 1100, equipado con una bomba cuaternaria, inyector automático $\mathrm{y}$ un detector de diodos en circuito integrado, acoplado a una estación de tratamiento de datos HP Chem Station (Rev. B. 04.01). La separación se llevó a cabo mediante una columna de fase reversa Spherisorb S3 ODS-2 C8, de 4,6 x $150 \mathrm{~mm}$ de dimensiones y un tamaño de partícula $3 \mu \mathrm{m}$, termostatizada a $35^{\circ} \mathrm{C}$. Las condiciones de HPLC y de MS fueron descritas previamente por Dueñas et al. (2015).

\subsubsection{Identificación y cuantificación de compuestos fenólicos}

La identificación de compuestos fenólicos por HPLC-DAD-MS se realizó mediante comparación de los tiempos de retención, espectros UV-visible en el caso de disponer de patrones comerciales. La identidad y confirmación de otros compuestos se realizó a partir de la relación masa/carga $(\mathrm{m} / \mathrm{z})$ de cada compuesto y sus patrones de fragmentación obtenidos por el espectrómetro de masas.

Para el análisis cuantitativo de los compuestos fenólicos identificados, se realizaron las curvas de calibrado a partir de patrones externos. Las rectas de calibrado de los compuestos se calcularon inyectando distintas concentraciones de la solución patrón, en las mismas condiciones cromatográficas que las muestras, realizándose la regresión lineal frente al área del pico obtenida en cada caso. 
MAGDALENA M. ${ }^{a}$ DELCOURT-GARCÍA; MARÍA TERESA ESCRIBANO-BAILÓN; MONTSERRAT DUEÑAS DETERMINACIÓN DE LA COMPOSICIÓN FENÓLICA EN GRANOS ANCESTRALES DE USO ALIMENTARIO. COMPARACIÓN DE MÉTODOS ANALÍTICOS Y DE ACTIVIDAD ANTIOXIDANTE

\subsection{Determinación de compuestos fenólicos totales}

La determinación espectrofotométrica de compuestos fenólicos totales se realizó con los métodos de Folin-Ciocalteu y Fast Blue BB (FBBB).

\subsubsection{Método Folin-Ciocalteu}

El método de Folin-Ciocalteu consiste en la reacción de los compuestos fenólicos con el reactivo Folin de color amarillo (mezcla de wolframato sódico y molibdato sódico en ácido fosfórico, ácido fosfomolibdotúngstico) en medio alcalino, dando una coloración azul intensa. Se siguió el método de Singlenton et al. (1965) con ligeras modificaciones.

\subsubsection{Método Fast Blue BB}

El método FBBB consiste en la reacción del grupo $(-\mathrm{OH})$ de los compuestos fenólicos con el radical diazonio $\left(-\mathrm{N}_{2}-\right)$ del reactivo Fast Blue BB. Como resultado de la sustitución electrofílica aromática, se forma un azo complejo. El método se optimizó en el laboratorio, siguiendo el método de Medina et al. (2011).

En ambos métodos los resultados se obtuvieron por interpolación de la absorbancia sobre una curva de calibrado obtenida con disoluciones de ácido gálico, independientemente de los compuestos fenólicos presentes en las muestras. Los resultados fueron expresados en $\mathrm{mg}$ de ácido gálico /g de muestra.

\subsection{Determinación de la actividad antioxidante}

\subsubsection{Método TEAC (Capacidad Antioxidante Equivalente de Trolox)}

El ensayo de la actividad antioxidante equivalente de Trolox (TEAC) mide la capacidad de un compuesto para captar el radical coloreado ABTS ${ }^{\bullet}$, en un medio acuoso, convirtiendo el mismo en un producto decolorado (Arts et al., 2003). El protocolo que se utilizó fue descrito por Re et al. (1999).

\subsubsection{Método FRAP (Poder Antioxidante Reductor del Hierro)}

El método FRAP se basa en la capacidad que presentan los compuestos antioxidantes presentes en la muestra para donar electrones y reducir el ion férrico a ferroso en medio acuoso ácido. Se siguió el método de Benzie y Strain (1996) con ligeras modificaciones.

Los resultados en ambos métodos fueron expresados como $\mu \mathrm{mol}$ TROLOX/g muestra.

Ediciones Universidad de Salamanca / @@ $\quad$ FarmaJournal, vol. 5, núm. 2 (2020), pp. 27-41 


\subsection{Análisis estadístico}

Los datos se expresan como la media \pm desviación estándar $( \pm \mathrm{SD})$ del triplicado correspondiente a cada muestra analizada.

El análisis estadístico se llevó a cabo usando el análisis de la varianza (ANOVA) por comparación de medias con el test de Tukey HSD con el fin de indicar las diferencias significativas con un valor de significación de un $\mathrm{p}<0,05$.

\section{Resultados y Discusión}

\subsection{Determinación de compuestos fenólicos totales en los granos ancestrales}

La Tabla 1 muestra el contenido en compuestos fenólicos totales (mg ácido gálico/g muestra) determinados espectrofotométricamente por los métodos Folin-Ciocalteu y FBBB. Los resultados muestran contenidos de compuestos fenólicos totales significativamente más altos con el método FBBB que con el método estándar Folin-Ciocalteu, con unos niveles mayores a los 90 minutos de reacción, exceptuando la espelta. Esta tendencia también fue observada por otros autores (López Froilán et al., 2018; Maives et al., 2015; Medina et al., 2011). Esto podría deberse a que en el método Folin-Ciocalteu otros compuestos presentes en la matriz pueden actuar como interferentes.

El trigo sarraceno presentó el mayor contenido de compuestos fenólicos en ambos métodos, presentando diferencias significativas con respecto a las otras muestras estudiadas, seguidas del teff marrón y blanco. La espelta mostró la concentración más baja en compuestos fenólicos totales en ambos métodos.

TABLA 1. Contenido en compuestos fenólicos totales (mg GAE/g muestra) por Folin-Ciocalteu y Fast Blue BB

\begin{tabular}{|c|c|c|c|c|}
\hline MÉTODO & Teff blanco & Teff marrón & Trigo sarraceno & Espelta \\
\hline Folin Ciocalteu & $9,87 \pm 0,24^{\mathrm{c}}{ }_{\mathrm{A}}$ & $8,55 \pm 0,24^{\mathrm{b}}{ }_{\mathrm{A}}$ & $32,60 \pm 0,46^{\mathrm{d}}{ }_{\mathrm{A}}$ & $3,27 \pm 0,16^{\mathrm{a}}{ }_{\mathrm{B}}$ \\
\hline FBBB $60_{\min }$ & $44,17 \pm 1,39^{\mathrm{b}}{ }_{\mathrm{B}}$ & $50,87 \pm 6,09^{\mathrm{c}}{ }_{\mathrm{B}}$ & $67,99 \pm 0,44^{\mathrm{d}}{ }_{\mathrm{B}}$ & $1,94 \pm 0,33^{\mathrm{a}}{ }_{\mathrm{A}}$ \\
\hline FBBB 90${ }_{\min }$ & $51,36 \pm 1,32^{\mathrm{b}}{ }_{\mathrm{C}}$ & $60,55 \pm 6,78^{\mathrm{c}}{ }_{\mathrm{C}}$ & $77,32 \pm 0,71^{\mathrm{d}}{ }_{\mathrm{C}}$ & $2,07 \pm 0,06^{\mathrm{a}}{ }_{\mathrm{A}}$ \\
\hline
\end{tabular}

Letras minúsculas diferentes dentro de cada fila indican diferencias significativas. Letras mayúsculas diferentes en una misma columna muestran diferencias significativas entre los métodos. 


\subsection{Caracterización y composición de compuestos fenólicos en los granos ancestrales}

Los compuestos fenólicos fueron identificados mediante HPLC-DAD-MS. En la Tabla 2 se recogen las características cromatográficas, espectrales de los compuestos fenólicos detectados, así como la composición de los compuestos fenólicos en las semillas estudiadas. Estas características nos permiten obtener información sobre la posible estructura de los compuestos, así como de la posible familia a la que pueden pertenecer. De esta manera, se consiguió la identificación de compuestos fenólicos pertenecientes a compuestos no flavonoideos (compuestos hidroxibenzoicos e hidroxicinámicos) y compuestos flavonoideos (flavonoles, flavonas, flavanoles).

\subsubsection{Teff blanco y teff marrón}

En ambas variedades de teff se identificaron un total de 20 compuestos (Tabla 2). El grupo de las flavonas fue la familia de compuestos fenólicos más abundante detectada en ambas muestras, principalmente por glucósidos de apigenina y luteolina, representando casi el $100 \%$ del total de compuestos fenólicos (Tabla $3)$, siendo los compuestos mayoritarios para el teff blanco Ap-6-C-glucósido6"-O-(6"'-O-vanilloil)hexósido (111,98 mg/g semilla) y el para el teff marrón, Lu-6-C-glucósido-7-O-hexósido (3490,04 mg/g semilla).

En la variedad blanca este grupo constituyó el 99,86\% respecto al total, destacando una mayoría derivados de apigenina $(95,75 \%)$, mientras que en la variedad marrón (100\%) fueron derivados de luteolina (86,61 \%).

En la variedad blanca este grupo constituyó el 99,86 \% respecto al total, destacando una mayoría derivados de apigenina $(95,75 \%)$, mientras que en la variedad marrón (100 \%) fueron derivados de luteolina (86,61 \%).

TABLA 2. Caracterización de compuestos fenólicos y composición de teff blanco, marrón, trigo sarraceno y trigo espelta ( $\mu \mathrm{g} / \mathrm{g}$ muestra)

(Ap:Apigenina; Lu:Luteolina)

\begin{tabular}{|c|c|c|c|c|c|c|}
\hline Pico & $\mathbf{t}_{\mathrm{R}}(\min )$ & $\lambda_{\max }$ & {$[\mathrm{M}-\mathrm{H}]^{-}$} & fragmentos & Identificación & $\mu \mathrm{g} / \mathrm{g}$ \\
\hline \multicolumn{7}{|c|}{ Teff blanco } \\
\hline 1 & 5,34 & 326 & 353 & $\begin{array}{c}191,179,173,161, \\
135\end{array}$ & $\begin{array}{c}\text { Ácido } \\
\text { 3-O-cafeoilquínico }\end{array}$ & $\mathrm{t}$ \\
\hline 2 & 5,92 & 336 & 755 & $\begin{array}{c}503,473,341,311, \\
282\end{array}$ & $\begin{array}{c}\text { Ap 8-C glucósido-O- } \\
\text { hexósido-O-hexósido }\end{array}$ & $\mathrm{t}$ \\
\hline 3 & 11,21 & 326 & 353 & $191,179,135$ & Ácido cafeoilquínico & $\mathrm{t}$ \\
\hline
\end{tabular}


MAGDALENA M. DELCOURT-GARCÍA; MARÍA TERESA ESCRIBANO-BAILÓN; MONTSERRAT DUEÑAS DETERMINACIÓN DE LA COMPOSICIÓN FENÓLICA EN GRANOS ANCESTRALES DE USO ALIMENTARIO. COMPARACIÓN DE MÉTODOS ANALÍTICOS Y DE ACTIVIDAD ANTIOXIDANTE

\begin{tabular}{|c|c|c|c|c|c|c|}
\hline Pico & $t_{R}(\min )$ & $\lambda_{\max }$ & {$[\mathrm{M}-\mathrm{H}]^{-}$} & fragmentos & Identificación & $\mu \mathrm{g} / \mathrm{g}$ \\
\hline 4 & 11,85 & 340 & 609 & $\begin{array}{c}447,357,327,299 \\
285\end{array}$ & $\begin{array}{l}\text { Lu 6-C-glucósido-O- } \\
\text { hexósido }\end{array}$ & $6,14 \pm 0,82$ \\
\hline 5 & 12,62 & 340 & 593 & $473,383,353,297$ & Ap-6,8-di-C-glucósido & $6,24 \pm 1,01$ \\
\hline 6 & 13,68 & 338 & 739 & $\begin{array}{c}575,457,341,311 \\
283\end{array}$ & $\begin{array}{l}\text { Ap-6-C-glucósido } \\
\text { 2"-O-ramnósido-6"-O- } \\
\text { hexósido }\end{array}$ & $3,43 \pm 0,38$ \\
\hline 7 & 14,85 & 322 & 577 & 311,28 & Ap-4’-O-rutinósido & $2,96 \pm 0,20$ \\
\hline 8 & 15,25 & 338 & 593 & $\begin{array}{c}473,431,341,311 \\
283\end{array}$ & $\begin{array}{l}\text { Ap-6-C-glucósido-7-O- } \\
\text { hexósido }\end{array}$ & $\begin{array}{l}83,80 \pm \\
9,75\end{array}$ \\
\hline 9 & 15,64 & 334 & 563 & $\begin{array}{c}473,443,383,353 \\
283 \\
\end{array}$ & $\begin{array}{l}\text { Ap-6-C-hexósido-8-C- } \\
\text { pentósido }\end{array}$ & $\begin{array}{c}21,63 \pm \\
1,82\end{array}$ \\
\hline 10 & 16,19 & 350 & 447 & $357,327,299,285$ & Lu-8-C-glucósido & $3,60 \pm 0,45$ \\
\hline 11 & 16,93 & 314 & 337 & $161,163,173,155$ & $\begin{array}{l}\text { Ácido 4-O-p- } \\
\text { cumaroilquínico }\end{array}$ & $0,62 \pm 0,21$ \\
\hline 12 & 16,94 & 338 & 563 & $\begin{array}{c}473,383,353,325 \\
297 \\
\end{array}$ & $\begin{array}{l}\text { Ap-6-C-pentósido-8-C- } \\
\text { glucósido }\end{array}$ & $6,79 \pm 0,26$ \\
\hline 13 & 17,93 & 336 & 593 & $\begin{array}{c}413,413,353,341 \\
323,311,283\end{array}$ & $\begin{array}{c}\text { Ap-6-C-hexósido-2"-O- } \\
\text { hexósido }\end{array}$ & $2,27 \pm 0,79$ \\
\hline 14 & 18,24 & 336 & 431 & $341,311,283$ & Ap-8-C-glucósido & $\begin{array}{c}109,57 \pm \\
13,53\end{array}$ \\
\hline 15 & 20,35 & 338 & 431 & $\begin{array}{c}413,341,323,311, \\
283\end{array}$ & Ap-6-C-glucósido & $9,52 \pm 0,79$ \\
\hline 16 & 21,58 & 336 & 445 & $325,297,282$ & $\begin{array}{l}\text { 7-O-metil-Ap-6-C- } \\
\text { glucósido }\end{array}$ & $7,10 \pm 0,45$ \\
\hline 17 & 22,40 & 352 & 759 & $\begin{array}{c}411,357,339,327 \\
299\end{array}$ & $\begin{array}{c}\text { Lu-6-C-glucósido-6”- } \\
\text { O-(6”-O-vanilloil) } \\
\text { hexósido }\end{array}$ & $8,92 \pm 0,19$ \\
\hline 18 & 23,82 & 336 & 577 & 311,269 & Ap-7-O-rutinósido & $\begin{array}{c}41,65 \pm \\
2,39 \\
\end{array}$ \\
\hline 19 & 26,15 & 334 & 743 & $\begin{array}{c}341,323,311,283 \\
269\end{array}$ & $\begin{array}{l}\text { Ap-6-C-glucósido- } \\
\text { 6”-O-(6"'-O-vanilloil) } \\
\text { hexósido }\end{array}$ & $\begin{array}{c}111,98 \pm \\
7,19\end{array}$ \\
\hline 20 & 29,82 & 336 & 515 & $455,323,293,281$ & $\begin{array}{c}\text { Ap-8-C- } \\
\text { diacetilglucósido }\end{array}$ & $\begin{array}{c}28,69 \pm \\
2,46\end{array}$ \\
\hline \multicolumn{7}{|c|}{ Teff marrón } \\
\hline 1 & 6,04 & 340 & 771 & $\begin{array}{c}609,447,357,327, \\
298\end{array}$ & $\begin{array}{l}\text { Lu-8-C-glucósido-O- } \\
\text { hexósido-O-hexósido }\end{array}$ & $\begin{array}{l}46,18 \pm \\
10,28\end{array}$ \\
\hline 2 & 7,17 & 350 & 771 & $\begin{array}{c}609,411,357,327 \\
285 \\
\end{array}$ & $\begin{array}{l}\text { Lu-6-C-glucósido-6"- } \\
\text { O-dihexósido }\end{array}$ & $\begin{array}{c}23,33 \pm \\
4,31 \\
\end{array}$ \\
\hline
\end{tabular}

Ediciones Universidad de Salamanca / @@® 
MAGDALENA M. DELCOURT-GARCÍA; MARÍA TERESA ESCRIBANO-BAILÓN; MONTSERRAT DUEÑAS DETERMINACIÓN DE LA COMPOSICIÓN FENÓLICA EN GRANOS ANCESTRALES DE USO ALIMENTARIO. COMPARACIÓN DE MÉTODOS ANALÍTICOS Y DE ACTIVIDAD ANTIOXIDANTE

\begin{tabular}{|c|c|c|c|c|c|c|}
\hline Pico & $t_{R}(\min )$ & $\lambda_{\max }$ & {$[\mathrm{M}-\mathrm{H}]^{-}$} & fragmentos & Identificación & $\mu \mathrm{g} / \mathrm{g}$ \\
\hline 3 & 9,64 & 346 & 755 & $\begin{array}{c}473,357,339,327 \\
309,298\end{array}$ & $\begin{array}{l}\text { Lu-6-C-glucósido-2"- } \\
\text { O-ramnósido-6"-O- } \\
\text { hexósido }\end{array}$ & $\begin{array}{c}97,46 \pm \\
2,15\end{array}$ \\
\hline 4 & 11,67 & 350 & 609 & $\begin{array}{c}447,357,327,299, \\
285\end{array}$ & $\begin{array}{l}\text { Lu-6-C-glucósido-7-O- } \\
\text { hexósido }\end{array}$ & $\begin{array}{c}3490,04 \pm \\
22,12\end{array}$ \\
\hline 5 & 12,65 & 348 & 755 & $447,357,327,299$ & $\begin{array}{l}\text { Lu-6-C-glucósido-7-O- } \\
\text { ramnosil-hexósido }\end{array}$ & $\begin{array}{c}386,07 \pm \\
10,79\end{array}$ \\
\hline 6 & 15,32 & 338 & 593 & $\begin{array}{c}473,431,341,311, \\
283\end{array}$ & $\begin{array}{l}\text { Ap-6-C-glucósido-7-O- } \\
\text { hexósido }\end{array}$ & $\begin{array}{c}482,74 \pm \\
1,51 \\
\end{array}$ \\
\hline 7 & 15,62 & 334 & 563 & $\begin{array}{c}473,443,383,353, \\
283 \\
\end{array}$ & $\begin{array}{l}\text { Ap-6-C-hexósido-8-C- } \\
\text { pentósido }\end{array}$ & $\begin{array}{c}423,71 \pm \\
4,61 \\
\end{array}$ \\
\hline 8 & 16,13 & 350 & 447 & $357,327,299,285$ & Lu-8-C-glucósido & $\begin{array}{c}764,00 \pm \\
39,47\end{array}$ \\
\hline 9 & 16,46 & 340 & 623 & $\begin{array}{c}371,341,327,313 \\
269\end{array}$ & $\begin{array}{l}\text { 7-O-metil-Lu- } \\
\text { 6-C-glucósido } \\
\text { 6"-O-hexósido }\end{array}$ & $\begin{array}{c}267,63 \pm \\
18,07\end{array}$ \\
\hline 10 & 16,96 & 338 & 563 & $\begin{array}{c}473,383,353,325, \\
297\end{array}$ & $\begin{array}{l}\text { Ap-6-C-pentósido-8-C- } \\
\text { glucósido }\end{array}$ & $\begin{array}{c}154,24 \pm \\
19,08\end{array}$ \\
\hline 11 & 17,29 & 352 & 461 & $371,341,313,298$ & $\begin{array}{l}\text { 7-O-metil-Lu-8-C- } \\
\text { glucósido }\end{array}$ & $\begin{array}{c}46,67 \pm \\
4,49 \\
\end{array}$ \\
\hline 12 & 18,33 & 336 & 431 & $341,311,283$ & Ap-8-C-glucósido & $\begin{array}{c}179,53 \pm \\
13,05 \\
\end{array}$ \\
\hline 13 & 19,17 & 350 & 489 & $\begin{array}{c}429,357,327,299, \\
285\end{array}$ & Lu-8-C-acetilglucósido & $\begin{array}{c}68,31 \pm \\
1,68\end{array}$ \\
\hline 14 & 20,07 & 344 & 593 & 285 & Lu-7-O-rutinósido & $\begin{array}{c}423,86 \pm \\
19,15\end{array}$ \\
\hline 15 & 20,60 & 348 & 789 & $\begin{array}{c}609,411,357,339 \\
327,285\end{array}$ & $\begin{array}{c}\text { Lu-6-C-glucósido-6”- } \\
\text { O-(6”-O-siringoil) } \\
\text { hexósido }\end{array}$ & $\begin{array}{c}401,57 \pm \\
16,18\end{array}$ \\
\hline 16 & 21,18 & 348 & 447 & 327,285 & Lu-7-O-glucósido & $\begin{array}{c}273,74 \pm \\
10,28 \\
\end{array}$ \\
\hline 17 & 22,32 & 352 & 759 & $\begin{array}{c}411,357,339,327 \\
299\end{array}$ & $\begin{array}{c}\text { Lu-6-C-glucósido-6”- } \\
\text { O-(6"'-O-vanilloil) } \\
\text { hexósido }\end{array}$ & $\begin{array}{c}1798,08 \pm \\
52,98\end{array}$ \\
\hline 18 & 23,27 & 334 & 729 & $\begin{array}{c}609,411,357,339 \\
327,299,285\end{array}$ & $\begin{array}{c}\text { Lu-6-C-glucósido- } \\
\text { 6”-O-(6”- }-\mathrm{-}-\mathrm{p}- \\
\text { hidroxibenzoil)hexósido }\end{array}$ & $\begin{array}{c}58,41 \pm \\
9,28\end{array}$ \\
\hline 19 & 24,29 & 352 & 489 & $\begin{array}{c}429,357,327,299, \\
285\end{array}$ & Lu-8-C-acetilglucósido & $\begin{array}{c}31,24 \pm \\
1,73\end{array}$ \\
\hline 20 & 26,2 & 350 & 531 & $\begin{array}{c}471,339,327,309, \\
297\end{array}$ & Lu-8-C-diacetilhexósido & $\begin{array}{c}43,22 \pm \\
1,20\end{array}$ \\
\hline
\end{tabular}

Ediciones Universidad de Salamanca / @@®

FarmaJournal, vol. 5, núm. 2 (2020), pp. 27-41 
MAGDALENA M. DELCOURT-GARCÍA; MARÍA TERESA ESCRIBANO-BAILÓN; MONTSERRAT DUEÑAS DETERMINACIÓN DE LA COMPOSICIÓN FENÓLICA EN GRANOS ANCESTRALES DE USO ALIMENTARIO. COMPARACIÓN DE MÉTODOS ANALÍTICOS Y DE ACTIVIDAD ANTIOXIDANTE

\begin{tabular}{|c|c|c|c|c|c|c|}
\hline Pico & $t_{R}(\min )$ & $\lambda_{\max }$ & {$[\mathrm{M}-\mathrm{H}]^{-}$} & fragmentos & Identificación & $\mu g / g$ \\
\hline \multicolumn{7}{|c|}{ Trigo sarraceno } \\
\hline 1 & 4,74 & 262,294 & 3115 & 153 & $\begin{array}{c}\text { Ácido protocatequico } \\
\text { hexósido }\end{array}$ & trazas \\
\hline 2 & 5,50 & 280 & 451 & 289 & (Epi)catequina hexósido & $\begin{array}{c}1634,82 \pm \\
293,00\end{array}$ \\
\hline 3 & 6,20 & 271 & 451 & 289 & (Epi)catequina hexósido & $\begin{array}{c}857,61 \pm \\
43,24\end{array}$ \\
\hline 4 & 6,68 & 280 & 451 & 289 & (Epi)catequina hexósido & $\begin{array}{c}760,28 \pm \\
72,57\end{array}$ \\
\hline 5 & 7,68 & 316 & 487 & 179,135 & $\begin{array}{l}\text { Cafeoil hexósido } \\
\text { ramnoside } \\
\text { (swertiamacroside) }\end{array}$ & $\begin{array}{c}2066,23 \pm \\
84,71\end{array}$ \\
\hline 6 & 7,91 & 279 & 289 & $245,151,137$ & Catequina & $\begin{array}{c}870,77 \pm \\
6,77\end{array}$ \\
\hline 7 & 9,03 & 278 & 577 & $451,425,407,289$ & Dímero PC & $\begin{array}{c}204,55 \pm \\
26,49\end{array}$ \\
\hline 8 & 11,09 & 274 & 289 & $245,151,137$ & Epicatequina & $\begin{array}{c}1136,60 \pm \\
61,10\end{array}$ \\
\hline 9 & 11,77 & 302 & 531 & 223 & $\begin{array}{c}\text { Sinapoil hexósido } \\
\text { rhamnoside }\end{array}$ & $\begin{array}{c}114,87 \pm \\
3,03 \\
\end{array}$ \\
\hline 10 & 13,95 & 318 & 781 & 473,179 & $\begin{array}{l}\text { Cafeoil dihexoside } \\
\text { rhamnoside pentoside }\end{array}$ & $\begin{array}{c}329,45 \pm \\
25,75\end{array}$ \\
\hline 11 & 14,63 & 302 & 531 & 223 & $\begin{array}{c}\text { Sinapoil hexósido } \\
\text { rhamnoside }\end{array}$ & $\begin{array}{c}884,17 \pm \\
0,04\end{array}$ \\
\hline 12 & 15,01 & 308 & 827 & $\begin{array}{c}665,503,485,341 \\
323,179\end{array}$ & Tricafeoil dihexósido & $\begin{array}{c}463,27 \pm \\
56,43\end{array}$ \\
\hline 13 & 17,69 & 330 & 515 & 179 & Dicaffeoil quínico & $\begin{array}{c}110,20 \pm \\
24,12\end{array}$ \\
\hline 14 & 18,02 & 274 & 441 & 289,169 & Epicatequina galato & $\begin{array}{c}352,19 \pm \\
3,18\end{array}$ \\
\hline 15 & 18,05 & - & 833 & $561,543,289,271$ & $\begin{array}{c}\text { Trímero ( } 2 \text { afzelechin }+1 \\
\text { epicatequina) }\end{array}$ & $\begin{array}{c}310,79 \pm \\
53,87\end{array}$ \\
\hline 16 & 19,62 & 354 & 609 & 301 & Quercetina rutinósido & $\begin{array}{c}2027,59 \pm \\
98,60\end{array}$ \\
\hline 17 & 20,14 & 306 & 795 & $487,325,179$ & $\begin{array}{l}\text { Cafeoil dihexoside } \\
\text { dirhamnoside }\end{array}$ & $\begin{array}{c}104,05 \pm \\
11,57\end{array}$ \\
\hline 18 & 20,35 & 274 & 727 & $561,455,289,271$ & $\begin{array}{c}\text { Epiafzelquina- } \\
\text { epicatequina-metil galato }\end{array}$ & $\begin{array}{c}590,21 \pm \\
40,81\end{array}$ \\
\hline 19 & 21,00 & 352 & 463 & 301 & Quercetina glucósido & trazas \\
\hline
\end{tabular}

Ediciones Universidad de Salamanca / @థ®ब

FarmaJournal, vol. 5, núm. 2 (2020), pp. 27-41 
MAGDALENA M. DELCOURT-GARCÍA; MARÍA TERESA ESCRIBANO-BAILÓN; MONTSERRAT DUEÑAS DETERMINACIÓN DE LA COMPOSICIÓN FENÓLICA EN GRANOS ANCESTRALES DE USO ALIMENTARIO. COMPARACIÓN DE MÉTODOS ANALÍTICOS Y DE ACTIVIDAD ANTIOXIDANTE

\begin{tabular}{|c|c|c|c|c|c|c|}
\hline Pico & $t_{R}(\min )$ & $\lambda_{\max }$ & {$[\mathrm{M}-\mathrm{H}]^{-}$} & fragmentos & Identificación & $\mu \mathrm{g} / \mathrm{g}$ \\
\hline 20 & 23,65 & 274 & 753 & 289 & $\begin{array}{c}\text { Epicatequina- } \\
\text { epicatequina-dimetil } \\
\text { galato }\end{array}$ & $\begin{array}{c}803,79 \pm \\
43,88\end{array}$ \\
\hline 21 & 24,72 & 318 & 447 & 301 & Quercetina rhamnoside & trazas \\
\hline 22 & 26,59 & 354 & 651 & 301 & $\begin{array}{c}\text { Quercetina } \\
\text { acylrutinosido }\end{array}$ & trazas \\
\hline 23 & 26,71 & 274 & 741 & $469,319,271$ & $\begin{array}{c}\text { Epiafzelquina- } \\
\text { epicatequina-O-di } \\
\text { metilgalato }\end{array}$ & $\begin{array}{c}2467,75 \pm \\
134,91\end{array}$ \\
\hline 24 & 27,37 & 352 & 651 & 301 & $\begin{array}{l}\text { Quercetina } \\
\text { acetilrutinosido }\end{array}$ & $\begin{array}{c}71,20 \pm \\
11,79 \\
\end{array}$ \\
\hline 25 & 29,51 & 270 & 469 & $319,271,125$ & $\begin{array}{c}\text { Epicatequina-O-3,4- } \\
\text { dimetilgalato }\end{array}$ & $\begin{array}{c}990,18 \pm \\
2,69\end{array}$ \\
\hline
\end{tabular}

\section{Trigo espelta}

\begin{tabular}{|c|c|c|c|c|c|c|}
\hline 1 & 5,13 & 260,296 & 153 & 109 & Ácido protocatéquico & $\begin{array}{c}949,00 \pm \\
83,10\end{array}$ \\
\hline 2 & 7,32 & 306 & 337 & $\begin{array}{c}191,73,163,155, \\
137\end{array}$ & $\begin{array}{c}\text { Ácido } \\
p \text {-coumaroilquínico }\end{array}$ & $\begin{array}{c}59,80 \pm \\
13,90\end{array}$ \\
\hline 3 & 11,5 & 324 & 179 & 135 & Ácido cafeico & $\begin{array}{c}183,10 \pm \\
32,50\end{array}$ \\
\hline 4 & 13,37 & 332 & 593 & $473,443,383,353$ & Ap- 6,8, di C-glucósido & $9,10 \pm 2,20$ \\
\hline 5 & 13,4 & 280 & 203 & - & Triptófano & \\
\hline 6 & 14,6 & 336 & 563 & $\begin{array}{c}503,473,443,413, \\
383,353,297\end{array}$ & $\begin{array}{c}\text { Ap-C-hexósido-O- } \\
\text { pentósido }\end{array}$ & $\begin{array}{c}552,40 \pm \\
25,90\end{array}$ \\
\hline 7 & 14,9 & 336 & 563 & $\begin{array}{c}503,473,443,413, \\
383,353,298\end{array}$ & $\begin{array}{c}\text { Ap-C-hexósido-O- } \\
\text { pentósido }\end{array}$ & $\begin{array}{c}772,20 \pm \\
42,80\end{array}$ \\
\hline 8 & 15,5 & 338 & 563 & $\begin{array}{c}473,443,413,383, \\
353,299\end{array}$ & $\begin{array}{c}\text { Ap-C-hexósido-O- } \\
\text { pentósido }\end{array}$ & $\begin{array}{c}435,20 \pm \\
22,10\end{array}$ \\
\hline 9 & 15,88 & 336 & 563 & $\begin{array}{c}503,473,443,413, \\
383,353,298\end{array}$ & $\begin{array}{c}\text { Ap-C-hexósido-O- } \\
\text { pentósido }\end{array}$ & $\begin{array}{c}2181,90 \pm \\
91,40\end{array}$ \\
\hline 10 & 18,02 & 340 & 593 & $503,413,383$ & $\begin{array}{c}\text { Ap-C-hexóside-O- } \\
\text { hexósido }\end{array}$ & trazas \\
\hline 11 & 18,3 & 346 & 533 & $\begin{array}{c}515,473,443,413, \\
383,353,325,297\end{array}$ & $\begin{array}{c}\text { Ap-6,8, di C-pentósido } \\
4,50 \pm 0,10\end{array}$ \\
\hline 12 & 19,02 & 348 & 533 & $\begin{array}{c}515,473,443,413, \\
383,353,325,297\end{array}$ & $\begin{array}{c}\text { Ap-6,8, di C-pentósido } \\
149\end{array}$ & $\begin{array}{c}52,00 \pm \\
5,60\end{array}$ \\
\hline 13 & 19,25 & 324 & 193 & Ácido ferúlico & $\begin{array}{c}302,40 \pm \\
38,80\end{array}$ \\
\hline 14 & 19,69 & 328 & 563 & $\begin{array}{c}473,383,353 \\
\text { pentósido }\end{array}$ & $\begin{array}{c}\text { Ap-C-hexósido-O- } \\
\text { trazas }\end{array}$ \\
\hline
\end{tabular}

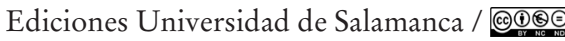


MAGDALENA M. DELCOURT-GARCÍA; MARÍA TERESA ESCRIBANO-BAILÓN; MONTSERRAT DUEÑAS DETERMINACIÓN DE LA COMPOSICIÓN FENÓLICA EN GRANOS ANCESTRALES DE USO ALIMENTARIO. COMPARACIÓN DE MÉTODOS ANALÍTICOS Y DE ACTIVIDAD ANTIOXIDANTE

\begin{tabular}{|c|c|c|c|c|c|c|}
\hline Pico & $\mathbf{t}_{\mathrm{R}}(\min )$ & $\lambda_{\max }$ & {$[\mathrm{M}-\mathrm{H}]^{-}$} & fragmentos & Identificación & $\mu \mathrm{g} / \mathrm{g}$ \\
\hline 15 & 20,77 & 352 & 639 & $435,373,331,315$ & Laricitrina O-rutinosido & trazas \\
\hline 16 & 21,7 & 332 & 769 & $\begin{array}{c}545,455,425,395, \\
365,335\end{array}$ & $\begin{array}{c}\text { Ap 2”-sinapoil-C- } \\
\text { hexoside-C-pentoside }\end{array}$ & $\begin{array}{c}132,40 \pm \\
20,80\end{array}$ \\
\hline 17 & 22,14 & 332 & 815 & $\begin{array}{c}739,545,345,330, \\
315\end{array}$ & $\begin{array}{c}\text { Siringetina dihexoside } \\
\text { rhamnoside }\end{array}$ & $\begin{array}{c}96,80 \pm \\
0,60\end{array}$ \\
\hline 18 & 22,69 & 332 & 769 & $\begin{array}{c}563,545,425,365, \\
335\end{array}$ & $\begin{array}{c}\text { Ap-2”-sinapoil-C- } \\
\text { hexósido-C-pentósido }\end{array}$ & $\begin{array}{c}377,00 \pm \\
52,80\end{array}$ \\
\hline 19 & 23,17 & 336 & 769 & $\begin{array}{c}563,545,455,425, \\
365,335\end{array}$ & $\begin{array}{c}\text { Api-2”-sinapoil-C- } \\
\text { hexósido-C-pentósido }\end{array}$ & $\begin{array}{c}235,20 \pm \\
11,60\end{array}$ \\
\hline 20 & 24,1 & 354 & 623 & 315 & $\begin{array}{c}\text { Isorhamnetina } \\
\text { rutinósido }\end{array}$ & $\begin{array}{c}53,50 \pm \\
3,20\end{array}$ \\
\hline 21 & 24,71 & 366 & 653 & 345,330 & $\begin{array}{c}\text { Siringetina rutinósido } \\
29,70 \pm \\
2,70\end{array}$ \\
\hline 22 & 25,45 & 344 & 769 & $\begin{array}{c}637,545,425,365, \\
335\end{array}$ & $\begin{array}{c}\text { Ap-2”-sinapoil-C- } \\
\text { hexósido-C-pentósido }\end{array}$ & $\begin{array}{c}41,80 \pm \\
5,40\end{array}$ \\
\hline
\end{tabular}

\subsubsection{Trigo sarraceno}

En el trigo sarraceno se identificaron un total de 25 compuestos (Tabla 2). El grupo de los flavanoles fue la familia de compuestos fenólicos más abundante, representando el 63,82\% (Tabla 3); como compuesto mayoritario destaca epiafzelquina-epicatequina-O-di metilgalato $(2467,75 \mathrm{mg} / \mathrm{g}$ semilla). En esta muestra se encontró el mayor contenido en compuestos hidroxicinámicos, un $23,83 \%$ con respecto al total.

\subsubsection{Trigo espelta}

El grupo de las flavonas fue la familia de compuestos fenólicos más abundante en el trigo espelta, representando el 75,15\% (Tabla 8); el compuesto mayoritario fue Ap-C-hexósido-O-pentósido (2181,90 mg/g semilla). Fue la única muestra que presentó compuestos no flavonoideos en forma de compuestos hidroxibenzóicos, representando casi un $15 \%$.

Con el objetivo de poder establecer una mejor comparación entre las muestras estudiadas, en la Tabla 3 se muestra el contenido de grupos de compuestos fenólicos para cada muestra (mg/g muestra) y el porcentaje de cada uno de los grupos con respecto al contenido total.

El contenido total más alto de compuestos fenólicos determinado por HPLC fue también en el trigo sarraceno $(17,08 \mathrm{mg} / \mathrm{g}$ muestra$)$. 
Respecto al contenido en flavonoides, el teff y la espelta mostraron como compuestos mayoritarios las flavonas (entre el 75 y el $100 \%$ del total). Sin embargo, en el trigo sarraceno los compuestos fenólicos mayoritarios fueron los flavanoles, representando un $63,82 \%$ del total.

TABla 3. Contenido de grupos de compuestos fenólicos para cada muestra (mg/g muestra) y composición porcentual de cada uno de los grupos con respecto al contenido total

\begin{tabular}{|c|c|c|c|c|}
\hline Grupos & Teff blanco & Teff marrón & Trigo sarraceno & Espelta \\
\hline $\begin{array}{c}\text { Compuestos } \\
\text { hidroxibenzoicos }\end{array}$ & $\mathrm{nd}$ & $\mathrm{nd}$ & trazas & $\begin{array}{c}0,95 \pm 0,08 \\
(14,94 \%)\end{array}$ \\
\hline $\begin{array}{c}\text { Compuestos } \\
\text { hidroxicinámicos }\end{array}$ & $\begin{array}{c}0,001 \pm 0,000^{\mathrm{a}} \\
(0,14 \%)\end{array}$ & $\mathrm{nd}^{\mathrm{a}}$ & $\begin{array}{c}4,07 \pm 0,16^{\mathrm{c}} \\
(23,83 \%)\end{array}$ & $\begin{array}{c}0,45 \pm 0,04^{\mathrm{b}} \\
(7,07 \%)\end{array}$ \\
\hline Flavonoles & $\mathrm{nd}^{\mathrm{a}}$ & $\mathrm{nd}^{\mathrm{a}}$ & $\begin{array}{c}2,10 \pm 0,11^{\mathrm{c}} \\
(12,29 \%)\end{array}$ & $\begin{array}{c}0,19 \pm 0,01^{\mathrm{b}} \\
(2,99 \%)\end{array}$ \\
\hline Flavonas & $\begin{array}{c}0,45 \pm 0,04^{\mathrm{b}} \\
(99,86 \%)\end{array}$ & $\begin{array}{c}9,45 \pm 0,05^{\mathrm{d}} \\
(100 \%)\end{array}$ & $\mathrm{nd}^{\mathrm{a}}$ & $\begin{array}{c}4,78 \pm 0,25^{\mathrm{c}} \\
(75,15 \%)\end{array}$ \\
\hline $\begin{array}{c}\text { Flavan 3-oles y } \\
\text { proantocianidinas }\end{array}$ & $\mathrm{nd}$ & $\mathrm{nd}$ & $\begin{array}{c}10,90 \pm 0,31 \\
(63,82 \%)\end{array}$ & $\mathrm{nd}$ \\
\hline $\begin{array}{c}\text { Total Compuestos } \\
\text { HPLC }\end{array}$ & $0,45 \pm 0,04^{\mathrm{a}}$ & $9,45 \pm 0,05^{\mathrm{c}}$ & $\begin{array}{c}17,08 \pm 0,04^{\mathrm{d}} \\
\mathbf{6}\end{array}$ & $\mathbf{6 , 3 6 \pm 0 , 3 6 ^ { \mathrm { b } }}$ \\
\hline
\end{tabular}

Letras diferentes dentro de cada fila indican diferencias significativas $(p<0,05)$.

En cuanto al contenido en ácidos fenólicos, identificamos compuestos hidroxibenzoicos e hidroxicinámicos. Los compuestos hidroxibenzoicos fueron principalmente cuantificados en las muestras de espelta representando un 14,94 $\%$. Sin embargo, los compuestos hidroxicinámicos se encuentran ampliamente representados en las muestras de trigo sarraceno suponiendo el $23,83 \%$ respecto al total; en la espelta representaron el 7,07 \% y en el teff blanco supusieron un porcentaje mínimo $(0,14 \%)$.

Como se puede observar, existen importantes diferencias cuantitativas en el contenido en compuestos fenólicos según el método utilizado. Hay que señalar que los métodos generales (Folin-Ciocalteu y FBBB) cuantifican según una recta de calibrado de ácido gálico, perteneciente a la familia de los ácidos hidroxibenzoicos, compuestos que, como hemos visto en la determinación por HPLC-DAD-MS, están escasamente representados en las muestras objeto de estudio. Sin embargo, en este último método, la cuantificación se lleva a cabo utilizando rectas de calibrado de patrones pertenecientes a las distintas familias de compuestos fenólicos analizados. 


\subsection{Determinación de actividad antioxidante (TEAC y FRAP)}

La muestra de trigo sarraceno presentó los valores más altos de actividad antioxidante para ambos métodos (valor TEAC 16,5 mmol Trolox/g muestra y valor FRAP 65,45 mmol Trolox/g muestra), mostrando diferencias significativas con respecto al resto. Sin embargo, el teff blanco y el teff marrón no mostraron diferencias significativas para ambos métodos. Valores TEAC que oscilaban entre 12,07 para el teff blanco y 11,35 mmol Trolox/g muestra para el teff marrón y los valores FRAP entre 14,26 para el teff blanco y 14,87 mmol Trolox/g muestra para el teff marrón. Las muestras de espelta fueron las que presentaron los valores más bajos para ambos métodos (valor TEAC 1,17 mmol Trolox/g muestra y valor FRAP 2, $60 \mathrm{mmol}$ Trolox/g muestra).

También se puede observar que existe una relación entre la mayor concentración de compuestos fenólicos encontrada en la muestra de trigo sarraceno con los valores más altos de la actividad antioxidante.

\section{Conclusión}

1. En general, los valores de compuestos fenólicos totales en los granos ancestrales estudiados, determinados por el método Fast Blue BB, han sido significativamente más altos que los calculados por el método estándar Folin-Ciocalteu y, en ambos casos, muy superiores a los determinados por HPLC-DAD-MS.

2. Según la composición fenólica determinada por HPLC-DAD-MS, parece más oportuno llevar a cabo la cuantificación de los compuestos fenólicos por este método que con los métodos espectrofotométricos generales, ya que estos últimos utilizan como recta de calibrado el ácido gálico, compuesto escaso o nulamente representado en las muestras analizadas.

3. Mediante la caracterización por HPLC-DAD-MS se ha conseguido la identificación de un gran número de compuestos fenólicos: no flavonoideos (compuestos hidroxibenzoicos e hidroxicinámicos) y flavonoideos (flavonoles, flavonas, flavanoles).

4. Las flavonas son la familia de compuestos fenólicos más abundante detectada en las muestras de espelta, teff, blanco y marrón. Sin embargo, en el trigo sarraceno son los flavanoles (64\%). De las flavonas presentes en el teff, parece que principalmente están en forma de glicósidos de apigenina en el blanco y de luteolina en el marrón; lo que indica diferencias importantes en sus rutas biosintéticas.

5. Los valores más elevados de actividad antioxidante, determinados mediante los métodos FRAP y TEAC, se han obtenido en las muestras de trigo 
sarraceno, mostrando diferencias significativas con respecto a las otras muestras, pudiéndose relacionar con su alto contenido en compuestos fenólicos.

\section{BibliografíA}

Arts Mariken JTJ, Dallinga JS, Voss HP, Haenen G, Bast A. A critical appraisal of the use of the antioxidant capacity (TEAC) assay in defining optimal antioxidant structures. Food Chem. 2003; 80(3):409-414.

Baye, K. Teff: Nutrient Composition and Health Benefits. International Food Policy Research Institute (IFPRI) and Ethiopian Development Research Institute (EDRI); 2014. p. 67.

Benincasa $\mathrm{P}$ et al. Phenolic compounds in grains, sprouts and wheatgrass of hulled and non-hulled wheat species. J Sci Food Agric. 2015; 95(9):1795-1803.

Benzie Iris FF, Strain JJ. The Ferric Reducing Ability of Plasma (FRAP) as a Measure of “Antioxidant Power": The FRAP Assay. Anal Biochem. 1996; 239(1):70-76.

Del Rio D, Rodríguez-Mateos A, Spencer JP, Tognolini M, Borges G, Crozier A. Dietary (poly)phenolics in human health: structures, bioavailability, and evidence of protective effects against chronic diseases. Antioxid Redox Signal. 2013; 18(14):1818-1892.

Dueñas M, Martínez-Villaluenga C, Limón RI, Peñas E, Frias J. Effect of germination and elicitation on phenolic composition and bioactivity of kidney beans. Food Res Int. 2015; 70:55-63.

Fraga CG, Kroft DC, Kennedy DO, Tomás-Berberán FA. The effects of polyphenols and other bioactives on human health. Food funct. 2019; 10:514-528.

Keriené I, Mankevičienė A, Bliznikas S, Jablonskyte-Rasce D, Maiksteniene S, Cesnuleviciene $\mathrm{R}$. Biologically active phenolic compounds in buckwheat, oats and winter spelt wheat. Zemdirbyste-Agriculture. 2015; 102(3):289-296.

López-Froilán R, Hernández-Ledesma B, Cámara M, Pérez-Rodríguez ML. Evaluation of the antioxidant potential of mixed fruit-based beverages: a new insight on the Folin Ciocalteu method. Food Anal Method. 2018; 11(10): 2897-2096.

Maives HA. Antioxidant phytochemicals of Hovenia dulcis Thunb. peduncles in different maturity stages. J Funct Food. 2015; 18(B):1117-1124.

Re R, Pellegrini N, Proteggente A, Pannala A, Yang M, Rice-Evans C. Antioxidant Activity Applying an improved ABTS radical cation decolorization Assay. Free Radic Biol Med. 1999; 26(9-10):1231-1237.

Sedeje I, Saka M, Mandi A, Mišan A, Tumbas V, Čanadanović-Brunet J. Buckwheat (Fagopyrum esculentum Moench) Grain and Fractions: Antioxidant Compounds and Activities. J Food Sci. 2012; 77(9):954-959.

Singleton V, Rossi J. Colorimetry of total phenolics with phosphomolybdic phosphotungstic acid reagents. Am. J. Enol. Vitic. 1965; 16:144-158. 
